University of New Hampshire

University of New Hampshire Scholars' Repository

Space Science Center

Institute for the Study of Earth, Oceans, and

Space (EOS)

2000

\title{
The TIGRE gamma-ray telescope
}

Terence J. O'Neill

University of California - Riverside

D Battacharya

University of California - Riverside

D Dixon

University of California - Riverside

M Polson

University of California - Riverside

R S. White

University of California - Riverside

See next page for additional authors

Follow this and additional works at: https://scholars.unh.edu/ssc

Part of the Astrophysics and Astronomy Commons

\section{Recommended Citation}

The TIGRE gamma-ray telescope O'Neill, T. J. and Bhattacharya, D. and Dixon, D. D. and Polson, M. and White, R. S. and Zych, A. D. and Ryan, J. and McConnell, M. and Macri, J. and Samimi, J. and Akyuz, A. and Mahoney, W. M. and Varnell, L., AIP Conference Proceedings, 510, 804-808 (2000), DOI:http://dx.doi.org/ 10.1063/1.1303309

This Conference Proceeding is brought to you for free and open access by the Institute for the Study of Earth, Oceans, and Space (EOS) at University of New Hampshire Scholars' Repository. It has been accepted for inclusion in Space Science Center by an authorized administrator of University of New Hampshire Scholars' Repository. For more information, please contact Scholarly.Communication@unh.edu. 


\section{Authors}

Terence J. O'Neill, D Battacharya, D Dixon, M Polson, R S. White, Allen Zych, James M. Ryan, Mark L. McConnell, John R. Macri, J Samimi, A Akyuz, W M. Mahoney, and I Varnell 


\section{AIP $\mid$ Proceedings}

\section{The TIGRE gamma-ray telescope}

T. J. O'Neill, D. Bhattacharya, D. D. Dixon, M. Polson, R. S. White, A. D. Zych, J. Ryan, M. McConnell, J. Macri, J. Samimi, A. Akyuz, W. M. Mahoney, and L. Varnell

Citation: AIP Conference Proceedings 510, 804 (2000); doi: 10.1063/1.1303309

View online: http://dx.doi.org/10.1063/1.1303309

View Table of Contents: http://scitation.aip.org/content/aip/proceeding/aipcp/510?ver=pdfcov

Published by the AIP Publishing

\section{Articles you may be interested in}

An Analysis Methodology for the Gammaray Large Area Space Telescope

AIP Conf. Proc. 707, 267 (2004); 10.1063/1.1751371

The TIGRE gamma-ray telescope

AIP Conf. Proc. 587, 882 (2001); 10.1063/1.1419515

Detection techniques of $\mu$ s gamma-ray bursts using ground-based telescopes

AIP Conf. Proc. 515, 253 (2000); 10.1063/1.1291376

LXeGRIT: The liquid xenon gamma-ray imaging telescope

AIP Conf. Proc. 510, 799 (2000); 10.1063/1.1303308

The IBIS Gamma-Ray telescope on INTEGRAL

AIP Conf. Proc. 510, 684 (2000); 10.1063/1.1303287 


\title{
The TIGRE Gamma-Ray Telescope
}

\author{
T.J. O'Neill ${ }^{1}$, D. Bhattacharya ${ }^{1}$, D.D. Dixon ${ }^{1}$, M. Polson ${ }^{1}$, R.S. \\ White $^{1}$, A.D. Zych ${ }^{1}$, J. Ryan ${ }^{2}$, M. McConnell' ${ }^{2}$, J. Macri' ${ }^{2}$, J. \\ $\mathrm{Samimi}^{3}$, A. Akyuz ${ }^{4}$,W.M. Mahoney ${ }^{5}$, L. Varnell ${ }^{5}$ \\ ${ }^{1}$ Institute of Geophysics and Planetary Physics, University of California, Riverside, CA \\ 92521, USA \\ ${ }^{2}$ Space Science Center, University of New Hampshire, Durham, NH 03824, USA \\ ${ }^{3}$ Dept. of Physics, Sharif University of Technology, Tehran, Iran \\ ${ }^{4}$ Dept. of Physics, Cukurova University, Adana, Turkey \\ ${ }^{5}$ Jet Propulsion Laboratory
}

\begin{abstract}
TIGRE is an advanced telescope for gamma-ray astronomy with a few arcmin resolution. From 0.3 to $10 \mathrm{MeV}$ it is a Compton telescope. Above 1 $\mathrm{MeV}$, its multi-layers of double sided silicon strip detectors allow for Compton recoil electron tracking and the unique determination for incident photon direction. From 10 to $100 \mathrm{MeV}$ the tracking feature is utilized for gamma-ray pair event reconstruction. Here we present TIGRE energy resolutions, background simulations and the development of the electronics readout system.
\end{abstract}

TIGRE Instrument Description: The TIGRE instrument [1] [2] [3], shown in Figure 1, features multilayers of silicon strip detectors (SSD) as both the Compton converter and recoil electron tracker. Double-sided SSDs provide submillimeter $\mathrm{x}$ and $\mathrm{y}$ spatial resolutions as the recoil electron is tracked through successive layers until it is fully absorbed. Position sensitive Ge and/or CdZnTe detectors are used as a calorimeter for the scattered photons and electron-positron pairs. $\mathrm{CsI}(\mathrm{Tl})$-Photodiode detector arrays on the bottom and sides serve as a low energy gamma-ray shield. This is important at energies below $1 \mathrm{MeV}$ where up-down discrimination of gamma-rays cannot be determined. The shield also serves as a Compton suppressor for gamma rays leaving the $\mathrm{Ge}$ and/or $\mathrm{CdZnTe}$. The $\mathrm{CsI}(\mathrm{Tl})$ arrays serve the dual purpose of a calorimeter for large scatter angle events as well as of a shield. This is particularly important for polarization measurements below $2 \mathrm{MeV}$. A particle anticoincidence plastic scintillator surrounds the entire sensitive material.

Gamma ray pair events are also detected in the traditional manner by tracking the electron and positron individually through successive layers of silicon strip detectors until at least one particle or annihilation photon exits and

CP510, The Fifth Compton Symposium, edited by M. L. McConnell and J. M. Ryan (c) 2000 American Institute of Physics 1-56396-932-7/00/\$17.00 


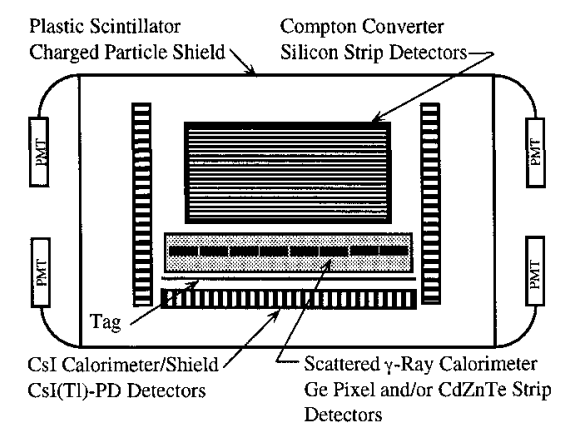

FIGURE 1. Baseline TIGRE instrument.

interacts in the $\mathrm{Ge} / \mathrm{CdZnTe}$ calorimeter. Both the energy losses and positions of the pair particles are measured in each Si layer as these particles are tracked through the array. For Compton events, where the electron direction is not apparent, we use the energy loss and position in each Si layer traversed to determine a Direction-of-Motion (DOM) parameter for each track. This parameter is similar to Time-of-Flight (TOF) that is used for up-down discrimination in conventional Compton telescopes.

Silicon Strip Detector Measurements: A mini TIGRE instrument was assembled using seven $4 \mathrm{~cm} \times 4 \mathrm{~cm}$ double-sided Si strip detectors and a single coaxial Ge detector. Figure 2 shows the laboratory setup for calibrating the

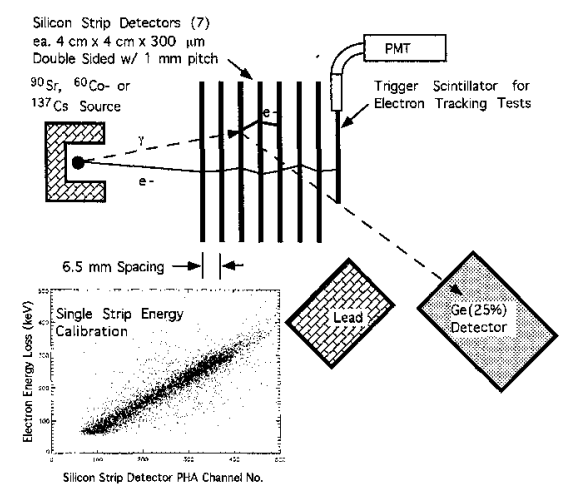

FIGURE 2. The laboratory setup used for the silicon germanium double scatter experiment. 


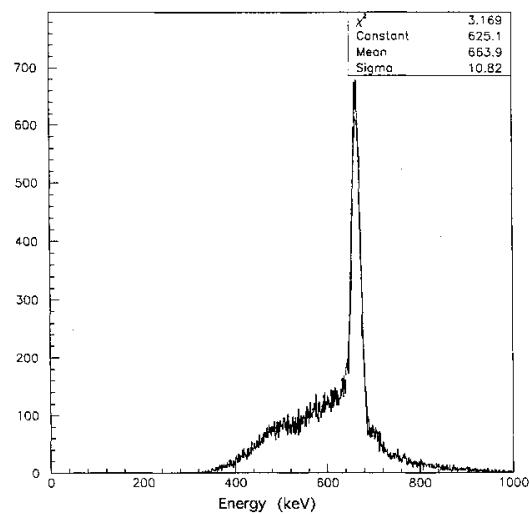

FIGURE 3. The spectrum of ${ }^{137} \mathrm{Cs}$ from the Si-Ge double scatter experiment.

Si detectors while the insert in the figure shows the calibration results for a single strip of a single detector. In a double scatter (Compton telescope) configuration, the wide range of scatter angles provides an equally wide range of energy losses in the Si. The true Si energy loss is determined from the measured Ge energy of the scattered gamma-ray and the source gamma-ray energy. This is compared to the measured Si energy thereby producing a calibration curve for each strip. A ${ }^{137} \mathrm{Cs}$ energy spectrum obtained by this method is shown in Figure 3. The measured energy resolution is $10.8 \mathrm{keV}$ $(1 \sigma)$ or $3.8 \%$. This resolution will improve with the new $10 \mathrm{~cm} \times 10 \mathrm{~cm} \mathrm{Si}$ detectors and low noise IDE-AS TA-1 chips with their trigger capability.

Figure 4 shows a nearly completed Si detector board containing a $10 \mathrm{~cm} \times$ $10 \mathrm{~cm}$ double-sided $300 \mu$ thick Si detector with one TA-1 hybrid wire-bonded to the 128 ohmic strips and one wire-bonded to the 128 junction strips. The hybrid consists of a custom ceramic carrier and a TA-1 chip. Figure 5 shows the shaper output signal (top curve) and the trigger for a test calibration pulse on a single channel. Figure 6 shows the gain, pedestal and noise for all 256 channels of the two TA-1 chips. The noise specification for this chip should allow us to achieve $1.1 \mathrm{keV}(1 \sigma, 318 \mathrm{ENC})$ energy resolution. For $0.5 \mathrm{MeV}$ electrons, where the total charge collected is $140,000 \mathrm{e}$ in three layers, the energy resolution should be $1.9 \mathrm{keV}(1 \sigma)$.

Simulation: We have modeled the TIGRE instrument described above with the general-purpose MCNP code developed at LANL. Energy resolutions for each individual $\mathrm{Si}$ and Ge detector element are taken as a conervative $3 \mathrm{keV}$ $(1 \sigma)$ and $1 \mathrm{keV}(1 \sigma)$, respectively. No CdZnTe was used in the simulation. The spatial resolutions are $0.75 \mathrm{~mm}$ and $2.0 \mathrm{~mm}$. Thresholds of $30 \mathrm{keV}$ for the $\mathrm{Si}$ and $\mathrm{Ge}$ detector elements are used. For the $\mathrm{CsI}(\mathrm{Tl})$ shield and plastic scintillators $100 \mathrm{keV}$ thresholds are used. For pair events, either the pair 


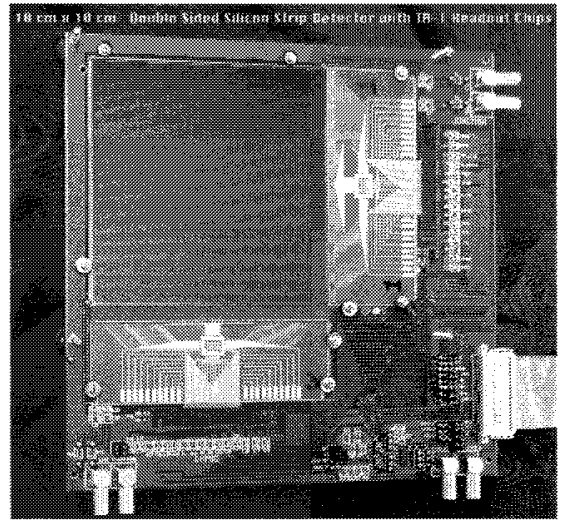

FIGURE 4. The double sided $10 \mathrm{~cm} \times 10 \mathrm{~cm}$ silicon strip detector with $2 \mathrm{TA}-1$ hybrid readout chips connected.

particle or an annihilation photon needs to be detected in the calorimeter. Event reconstruction is used to identify gamma-ray with directions within the instrument's FOV. The TIGRE angular resolutions and sensitivities are presented in [4].

TIGRE has a broad maximum efficiency of $5 \%$ and a high effective area of $80 \mathrm{~cm}^{2}$ in the Compton regime above $0.5 \mathrm{MeV}$. All Compton events are either "tracked" or "non-tracked" depending on the number of silicon layers the recoil electron traverses. The percentage of tracked events increase from $50 \%$ at $0.5 \mathrm{MeV}$ to $>90 \%$ above $2 \mathrm{MeV}$. In the pair regime the efficiency remains constant with a value of $5 \%$ up to $100 \mathrm{MeV}$. The effective combination of shielding and kinematics reduces the background contribution from outside the FOV $(\sim \pi \mathrm{sr})$. Figure 7 shows the relative efficiencies at $1.8 \mathrm{MeV}$ for an isotropic background as a function of the incident zenith angles for cases of no shielding, shielding and shielding with the tracking cuts.

\section{REFERENCES}

1. O'Neill, T. J. et al. 1995, IEEE Trans. Nuc. Sci., 42, 933.

2. Akyuz A. et al. 1995, Experimental Astronomy, 6, 274.

3. Tumer et al. 1995, IEEE Trans. Nuc. Sci., 42, No. 4, 933.

4. Bhattacharya, D. et al. 1999, 26th ICRC Conf. Proc., 5, 72. 


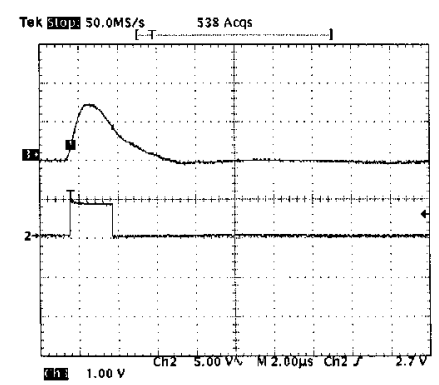

FIGURE 5. The TA-1 shaper output signal (top curve) and the trigger for a test calibration pulse. The trigger is necessary to eliminate accidental events.

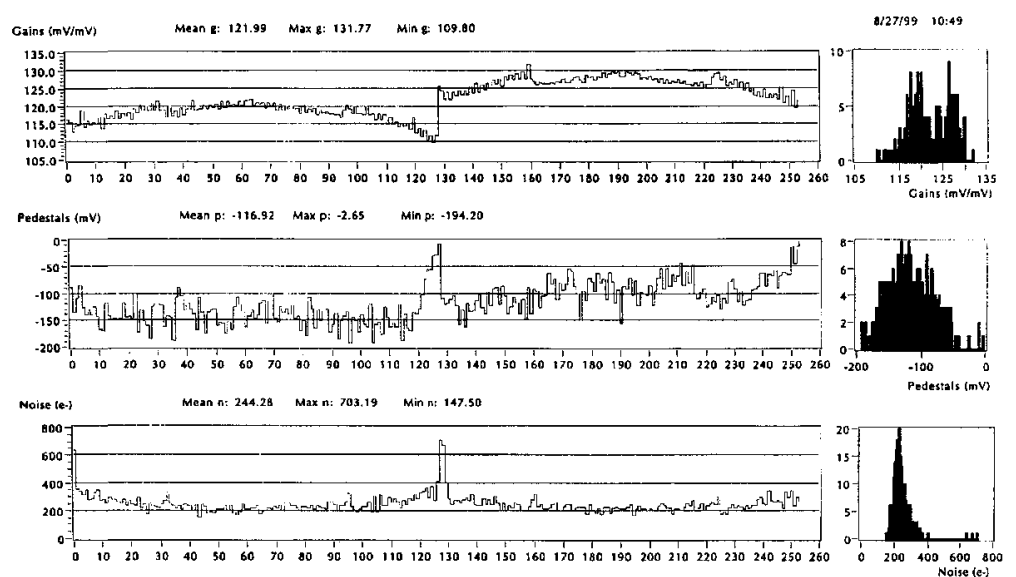

FIGURE 6. The preliminary gain, pedestal and noise performance for two 128 channel TA-1 chips serially connected.

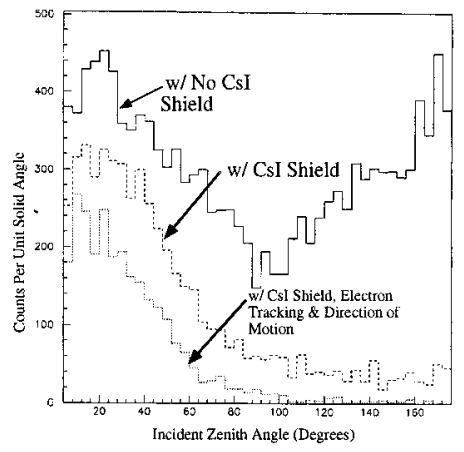

FIGURE 7. Relative efficiencies at $1.8 \mathrm{MeV}$ for an isotropic background as a function of the incident zenith angles for cases of no shielding, shielding and shielding with the tracking cuts. 